\title{
P-ONE second pathfinder mission: STRAW-b
}

\section{Immacolata Carmen Rea ${ }^{a, 1, *}$ Kilian Holzapfel $^{a}$ and Andrew Baron ${ }^{b}$ on behalf of the P-ONE Collaboration \\ (a complete list of authors can be found at the end of the proceedings)}

${ }^{a}$ Physic Dept., Technical University Munich, James-Franck-Str. 1, 85748 Garching, Germany

${ }^{b}$ Ocean Networks Canada, Victoria, Canada

E-mail: imma.rea@tum.de, kilian.holzapfel@tum.de

The Pacific Ocean Neutrino Explorer (P-ONE) [1] collaboration was born to build a new large-scale neutrino telescope in the Pacific Ocean, at $2600 \mathrm{~m}$ b.s.l. in Cascadia Basin, off Vancouver Island. The first steps aimed at the feasibility study and the characterisation of the optical properties of the site with a first pathfinder project named STRAW (STRing for Absorption length in Water) [2] [3] [4], deployed in 2018. During the last two years, a second pathfinder project has been developed: STRAW-b. The main goal of STRAW-b is to validate the attenuation length already measured by STRAW and to add new information on the background characterisation with the study of the deep sea diffused light spectrum. It consists of a $500 \mathrm{~m}$ mooring (electrical-optical cable communication) equipped with three Standard Modules for environmental monitoring and seven Specialised Modules for background analysis and attenuation length measurements. All the modules are hosted in spherical 13" high-pressure resistant glass housings. Its design started at the end of 2018 and after about two years it has been successfully deployed in summer 2020 in Cascadia Basin site, connected to the underwater Ocean Networks Canada infrastructure about 40 meters away from STRAW. We present all the steps from the design to the realisation of the mooring, with a special focus on the adopted technologies.

\footnotetext{
*** 37th International Cosmic Ray Conference (ICRC2021), ***

*** 12-23 July $2021 * * *$

*** Berlin, Germany - Online ***
}

\footnotetext{
${ }^{*}$ Presenter
} 


\section{Introduction}

After the successful deployment of STRAW and its promising first results, the feasibility study of P-ONE continued with a second milestone: the STRAW-B pathfinder.

The scientific goal of STRAW-b was a complete characterisation of the Cascadia Basin site. In particular, we focused on the monitoring of the light background sources and on complementing the measurement of the water attenuation length done by STRAW.

STRAW-b has been deployed during summer 2020, after an intense preparatory phase of about two years. The mooring is connected to the underwater Ocean Networks Canada infrastructure, $\sim 40$ meters from STRAW fig. 1.

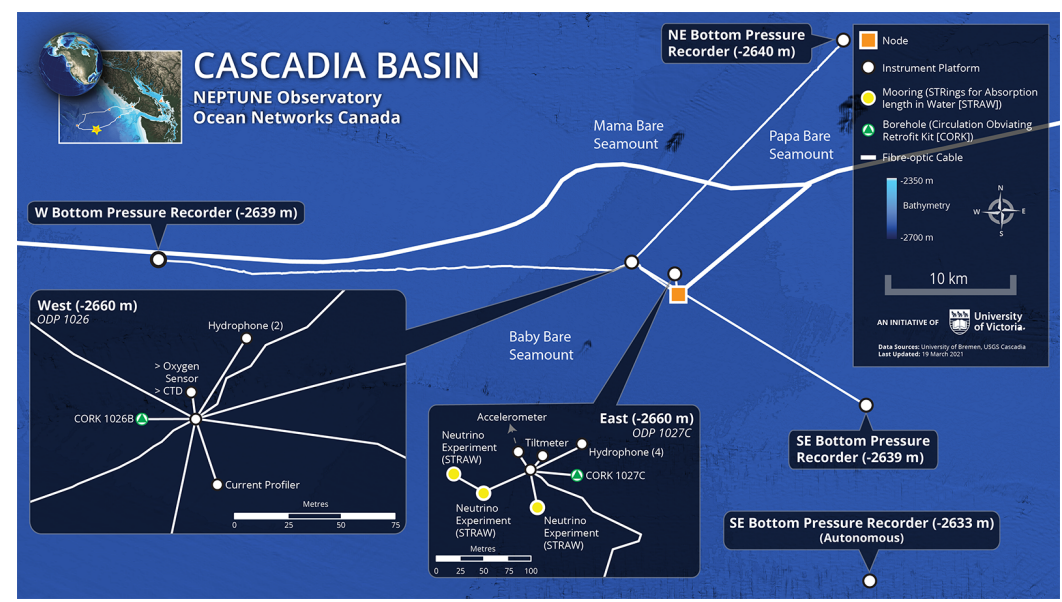

Figure 1: Map of the positions for STRAW and STRAW-b in Cascadia Basin.

\section{Design}

STRAW-b is a $500 \mathrm{~m}$ long mooring, equipped with ten modules (three Standard Modules and seven Specialised Modules), all housed in spherical 13" high-pressure resistant glass spheres (except for the WOM module), see fig. 2.

The aim of the Standard Modules is to monitor the environmental conditions (pressure, temperature, humidity) and reconstruct the position of all the modules, using magnetic field sensors and accelerometers. Their inner mechanical and electronic structure is the base for the Specialised Modules.

The Specialised Modules have been designed with the specific purpose of achieving a full characterisation of the water optical properties and light background of the Cascadia Basin site, with a special focus on the bioluminescence emission spectrum. The Specialised Modules are two LiDARs (Light Detection And Ranging), one muon tracker, two PMT-based spectrometers, one mini spectrometer and one Wavelength shifting Optical Module (WOM).

As can be seen in fig. 3, the ten modules are distributed from $120 \mathrm{~m}$ to $432 \mathrm{~m}$, equally spaced along the mooring, that is made of a bundle of ten Vertical Electrical Optical Cables - VEOCs. The VEOCs are hybrid cables composed of two copper wires, used for the power distribution, and two single-mode fibres for data transmission. The VEOCs are coupled to the mechanical backbone, 


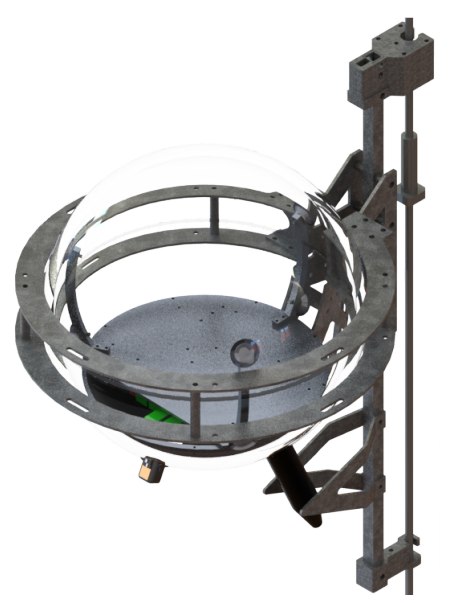

Figure 2: Schematic of STRAWb 13" high-pressure resistant glass housing and its mounting. Picture courtesy of Christian Spannfellner, TUM.

made of a stainless steel spiral rope with a diameter of $8.4 \mathrm{~mm}$. To reduce light reflection and avoid corrosion from the saltwater, it has been coated with a Galvan zinc-aluminium alloy.

In the upper part, a system of two 44" buoys, with a combined buoyancy of $622 \mathrm{~kg}$ (depth rating of $2500 \mathrm{~m}$ ), is connected to the mooring with a swivel. In this way, the line can rotate freely with currents without being damaged.

The VEOCs are connected to the submarine network through a mini junction box, developed and built by the Ocean Networks Canada team. In the mini junction box, the high voltage coming from the node is converted to lower voltages $(15 \mathrm{~V}, 24 \mathrm{~V}, 48 \mathrm{~V}$ up to $96 \mathrm{~V})$ and distributed to the modules. In addition, it is responsible for communication with all the deployed instruments. Similar to the STRAW anchor, the STRAW-b anchor is made by two train wheels (designed by ONC) and weighs approximately $592 \mathrm{~kg}$ in water.

\subsection{Standard Modules}

The Standard Modules are aimed at providing constant monitoring of environmental parameters like pressure, temperature and humidity. As for all the other STRAW-b modules, the housing is a 13" glass sphere, made by N-BK7 borosilicate glass with a transmissivity $>95 \%$ in the range between $350 \mathrm{~nm}$ and $600 \mathrm{~nm}$. The spheres are made by two hemispheres, closed by a glass-onglass contact with an internal under-pressure of $\sim 0.3$ bar and sealed by several layers of rubber edge protection and adhesive tapes at the equator. A built-in electro-optical penetrator allows the connection with the VEOC.

The readout electronics and the internal mountings fit a minimum space inside the sphere. The boards are mounted on a laser-cut aluminium plate, which is connected to on an aluminium bowl. The latter is in direct contact with the lower glass hemisphere, serving heat dissipation purposes. The stability of the mechanical structure is enhanced by three strives (whose shape follows the radius of the sphere), mounting with attached suction cups. The internal mounting is secured in place by a spring-loaded configuration, established by the suction cups [7], fig. 4 . 


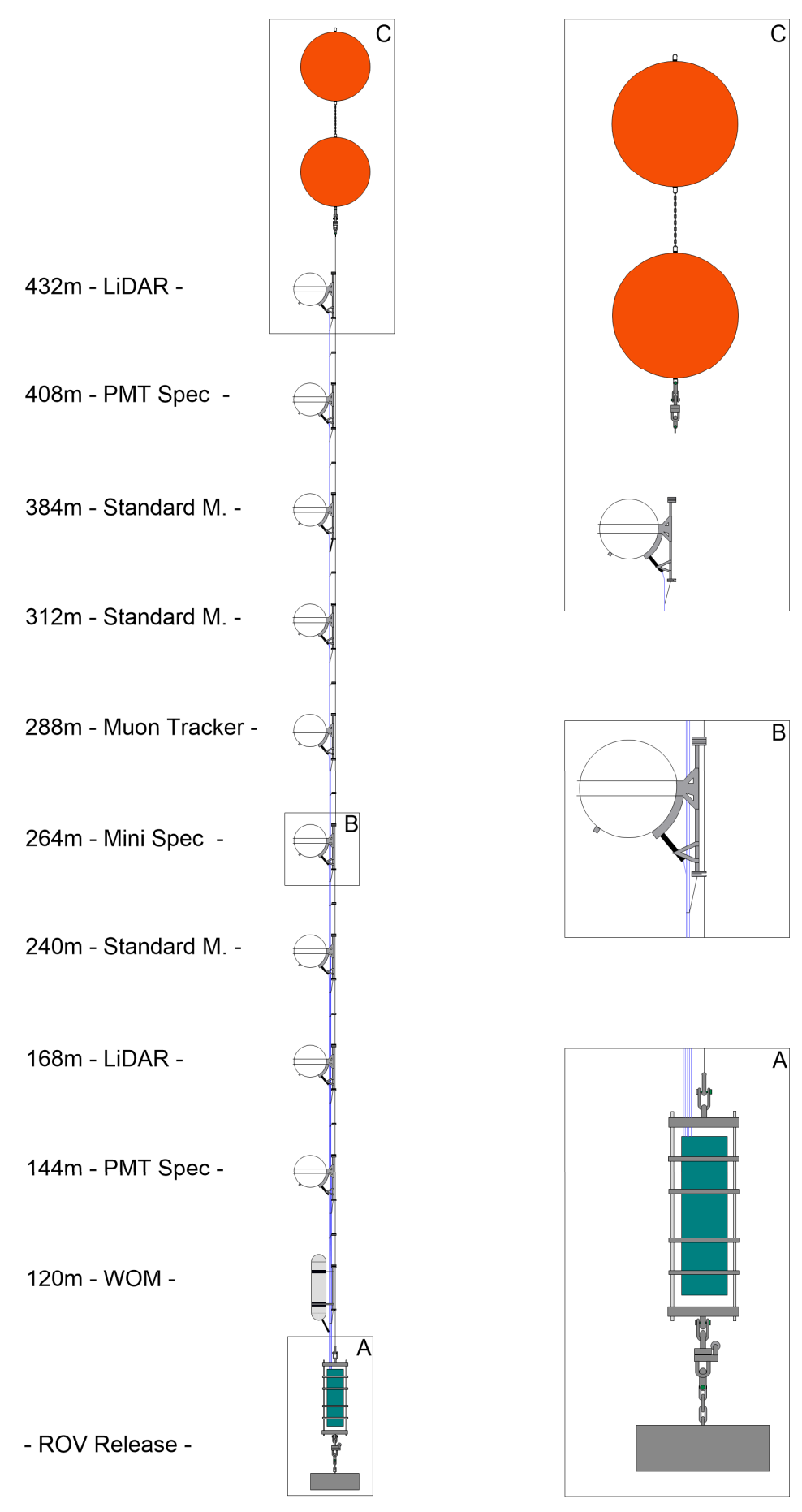

Figure 3: Sketch of the STRAW-b mooring: a bundle of 10 VEOCs equipped with modules coupled to the mechanical backbone made of steel cable. On the right of the picture, a detailed view of the two buoys system and mini junction box with the anchor. Picture courtesy of Christian Spannfellner, TUM. 


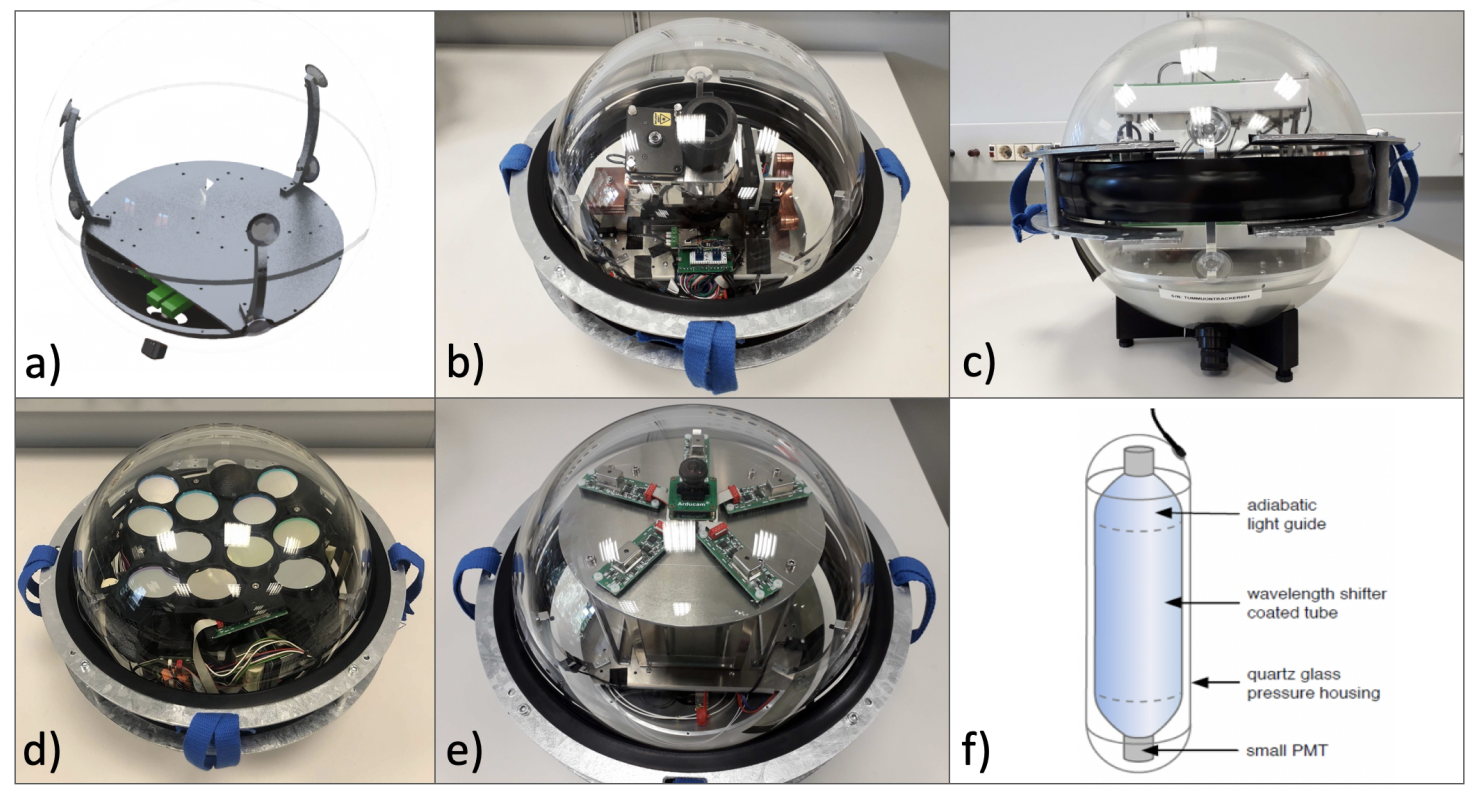

Figure 4: The STRAW-b modules. a) the Standard Module internal mechanics. The complete mechanical substructure with enclosed readout electronics is located $75 \mathrm{~mm}$ below the sphere equator. Pictures courtesy of Christian Spannfellner, TUM. b) Frontal picture of one of the two LiDAR modules. c) The Muon Tracker Module. d) one of the two PMT spectrometer modules. e) The Minispectrometer Module. f) The Wavelength Shifting Optical Module, picture courtesy of JGU Mainz group.

\subsection{Specialised Modules}

The Specialised Modules are hosted in optically enhanced glass upper hemispheres, to maximize the transmittance of light.

\subsubsection{The LiDAR Module}

The two LiDAR (Light Detection and Ranging) modules, fig. 4, aim at complementing and improving the measurement of the attenuation length done by STRAW, discriminating between scatterings and absorptions.

A laser diode emits a sequence of $10 \mathrm{~ns}$ light pulses at $450 \mathrm{~nm}$ into the seawater. Such pulses are successively scattered back and detected. The optical receiving system is made by a lens, equipped with a narrow-band filter, that focuses the light into a $\mu$ PMT. In addition, the LiDAR modules host two ultra-bright, downward-pointing LEDs (broad-spectrum white LED and UV LED) to calibrate the two PMT-spectrometers placed directly below them.

\subsubsection{The Muon Tracker Module}

In an underwater neutrino telescope, atmospheric muons represent one of the main sources of background.

The muon flux in the deep ocean has been well studied by different underwater experiments. The STRAW-b Muon Tracker, fig. 4, aims to confirm these measurements in the deep Pacific Ocean. The module is made by two scintillator planes, coupled with optical gel to arrays of SiPMs. Only time coincident signals in the two different scintillator planes are counted as muon events [6]. 


\subsubsection{The PMT spectrometer module}

An optical module capable of measuring the spectrum of incoming light can add new discriminating constraints in the background analysis and provide useful information about the bioluminescent burst emission mechanisms.

With STRAW, we have performed long-term monitoring of the optical background. However, the measurement of the light spectrum in-situ has never been achieved by any neutrino telescope so far. This is the science goal of the two STRAW-b PMT spectrometer modules, fig. 4. The module is made of twelve PMTs, each coupled to a focusing lens and a narrow-band filter (nominal FWHM from 10 to $50 \mathrm{~nm}$ ). The total range goes from 350 to $550 \mathrm{~nm}$. In addition, a compact camera is mounted at the centre of the module, to take pictures of bright bioluminescence events [5].

\subsubsection{The Mini spectrometer Module}

The Mini spectrometer module aims to complement the measurements of the PMT spectrometer. In particular, it is optimised for high-intensity bioluminescence bursts, for which the PMT spectrometer is expected to saturate. The module is composed of 5 Hamamatsu mini spectrometers (C12880MA), fig. 4, very compact and hermetic for high reliability against humidity. Each mini spectrometer has a high-sensitivity CMOS linear image sensor, composed of 288 pixels. The spectral range response goes from 340 to $850 \mathrm{~nm}$, with a spectral resolution of $15 \mathrm{~nm}$. Also, in this case, a compact astronomy camera for bioluminescence events is installed at the centre of the module.

\subsubsection{The Wavelength Shifting Optical Module (WOM)}

The Wavelength shifting Optical Module (WOM), fig. 4 has been developed for the IceCube Upgrade by the Johannes Gutenberg University of Mainz, Germany. Differently from all the optical modules in neutrino experiments, it is optimized for the detection of UV photons. An external quartz cylindrical high-pressure resistant housing hosts a smaller cylinder, coated with a wavelength shifting optical paint. The incident UV photons are absorbed and re-emitted in the visible range. Then, they can be detected by two PMTs coupled to the internal cylinder with an optical glue. In this way, an unexplored deep-sea background light wavelength region can be investigated.

To check the functionalities of the sensors, the electronics of the muon tracker, LiDARs and PMT-spectrometers have been provided with blue, white and UV LEDs looking downwards.

\section{Deployment}

The deployment strategy adopted by STRAW-b followed the top-down approach, similar to STRAW. The buoys were deployed first and dragged far from the ship by a Rigid Inflatable Boat (RIB). Then, the crew members from the Ocean Networks Canada team coupled the two structures that composed the string (the VEOCs and the steel cable), while attaching the modules to the string on the back deck of the ship before laying the string on the water surface, fig. 5. After the deployment, to check if the modules showed some visible damage potentially occurred during the descent, the ROV camera performed a very close visual inspection of the entire string, fig. 6 . 


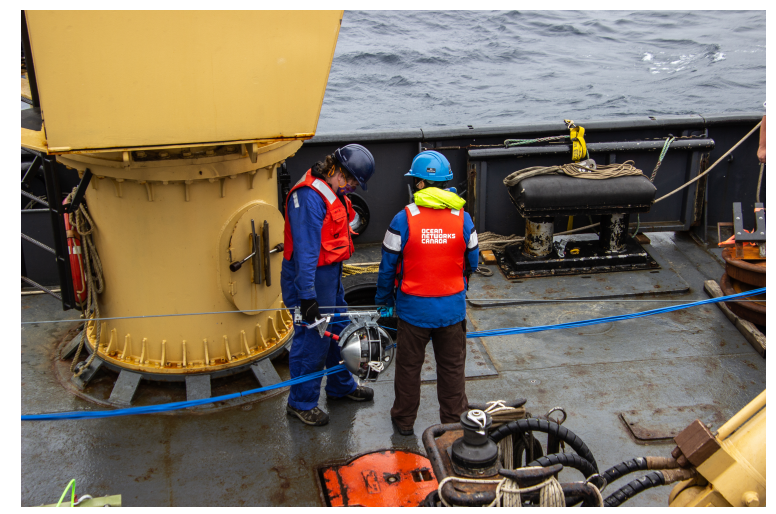

Figure 5: Ocean Networks Canada team during deployment operation.

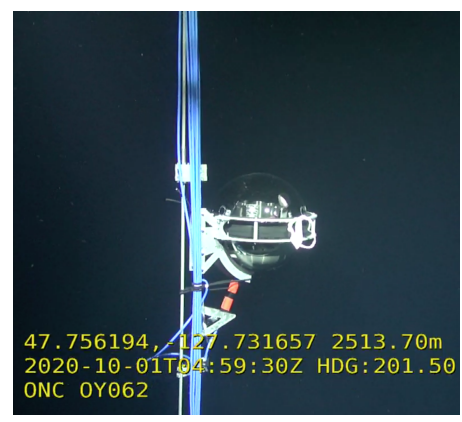

Figure 6: Picture taken from the live stream video transmitted during the visual inspection of the ROV.

\section{Current Status}

The STRAW-b modules are taking data continuously for several months, and the acquisition software is under finetuning process to guarantee stability over time. Moreover, the integration into publicly available ONC's database Oceans 2.0 (https://data.oceannetworks.ca) process is finished. Currently, the focus is on analysing the data of the various sensors. In fig. 7 and 8 , we show a camera image and the PMT spectrometer raw data belonging to the same bioluminescence emission event.

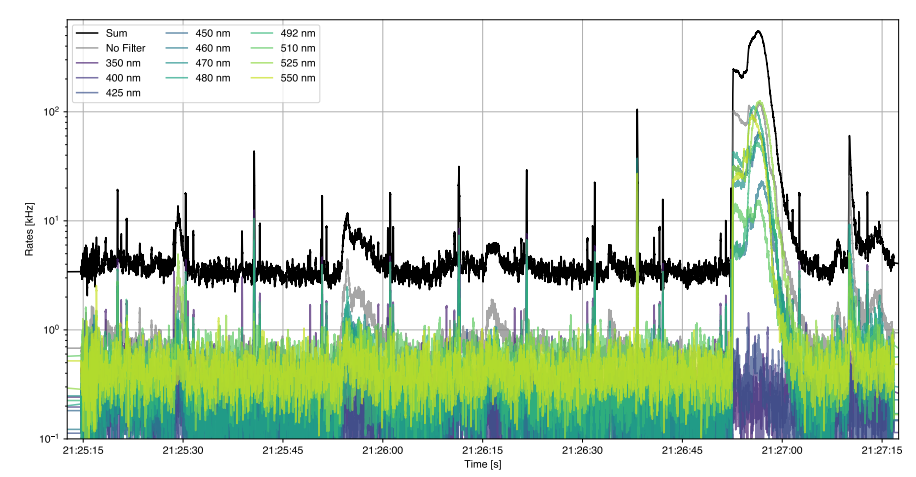

Figure 7: Bioluminescence event detected by the PMT-spectrometer module. 


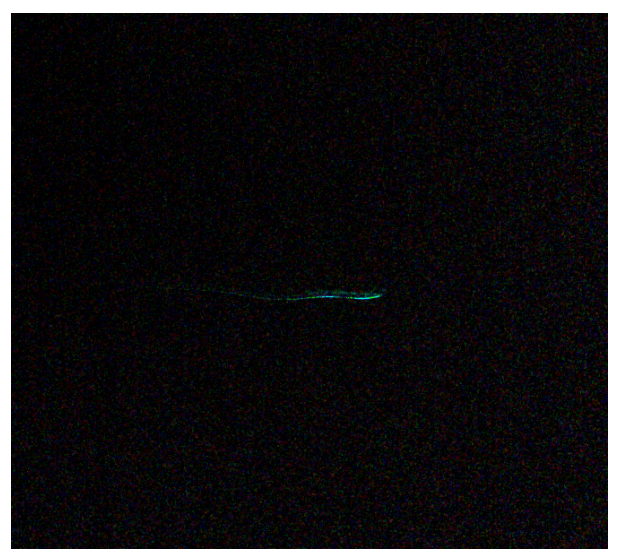

Figure 8: Bioluminescence event detected by the camera of the PMT-spectrometer module.

\section{References}

[1] Agostini, M., Böhmer, M., Bosma, J. et al. The Pacific Ocean Neutrino Experiment, Nat Astron 4, 913-915 (2020).

[2] Fruck, Christian and Rea, Immacolata Carmen STRAW: STRings for Absorption length in Water, PoS ICRC2019, 890 (2019).

[3] Rea, Immacolata Carmen et al. STRAW-Strings for Absorption Length in Water Pathfinder for a Potential New Neutrino Telescope Site in the Pacific Ocean, JPS Conf. Proc. 27011016 (2019).

[4] M., Böhmer et al. STRAW (STRings for Absorption length in Water): pathfinder for a neutrino telescope in the deep Pacific Ocean, JINST14 P02013 (2019)

[5] Li Ruohan Development of STRAW-b: Strings for Absorption length in Water for Future Neutrino Telescope in Deep Pacific Ocean, 2020

[6] Eva Laura Winter Muon Tracker for the Second Pathfinder of the Pacific Ocean Neutrino Explorer 2019

[7] Christian Spannfellner Development of pathfinder missions and instruments for the Pacific Ocean Neutrino Experiment 2020 


\section{Full Authors List: P-ONE Collaboration}

Nicolai Bailly ${ }^{1}$, Jeannette Bedard ${ }^{1}$, Michael Böhmer ${ }^{2}$, Jeff Bosma ${ }^{1}$, Dirk Brussow ${ }^{1}$, Jonathan Cheng ${ }^{1}$, Ken Clark ${ }^{3}$, Beckey Croteau ${ }^{1}$, Matthias Danninger ${ }^{4}$, Fabio De Leo ${ }^{1}$, Nathan Deis ${ }^{1}$, Matthew Ens $^{4}$, Rowan Fox ${ }^{1}$, Christian Fruck ${ }^{2}$, Andreas Gärtner ${ }^{5}$, Roman Gernhäuser $^{2}$, Darren Grant ${ }^{6}$, Helen He ${ }^{1}$, Felix Henningsen ${ }^{7}$, Kilian Holzapfel ${ }^{2}$, Ryan Hotte ${ }^{1}$, Reyna Jenkyns ${ }^{1}$, Hamish Johnson ${ }^{4}$, Akanksha Katil ${ }^{5}$, Claudio Kopper ${ }^{6}$, Carsten B. Krauss ${ }^{5}$, Ian Kulin ${ }^{1}$, Klaus Leismüller ${ }^{2}$, Sally Leys ${ }^{8}$, Tony Lin ${ }^{1}$, Paul Macoun ${ }^{1}$, Thomas McElroy $^{5}$, Stephan Meighen-Berger ${ }^{2}$, Jan Michel ${ }^{9}$, Roger Moore ${ }^{5}$, Mike Morley ${ }^{1}$, Laszlo Papp ${ }^{2}$, Benoit Pirenne ${ }^{1}$, Tom Qiu ${ }^{1}$, Mark Rankin ${ }^{1}$, Immacolata Carmen Rea ${ }^{2}$, Elisa Resconi ${ }^{2}$, Adrian Round ${ }^{1}$, Albert Ruskey ${ }^{1}$, Ryan Rutley ${ }^{1}$, Christian Spannfellner ${ }^{2}$, Jakub Stacho $^{4}$, Ross Timmerman ${ }^{1}$, Meghan Tomlin ${ }^{1}$, Matt Tradewell ${ }^{1}$, Michael Traxler ${ }^{10}$, Matt Uganecz ${ }^{1}$, Seann Wagner ${ }^{1}$, Juan Pablo Yañez ${ }^{5}$, Yinsong Zheng ${ }^{1}$

${ }^{1}$ Ocean Networks Canada, University of Victoria, Victoria, British Columbia, Canada.

${ }^{2}$ Department of Physics, Technical University of Munich, Garching, Germany.

${ }^{3}$ Department of Physics, Engineering Physics and Astronomy, Queen?s University, Kingston, Ontario, Canada.

${ }^{4}$ Department of Physics, Simon Fraser University, Burnaby, British Columbia, Canada.

${ }^{5}$ Department of Physics, University of Alberta, Edmonton, Alberta, Canada.

${ }^{6}$ Department of Physics and Astronomy, Michigan State University, East Lansing, MI, USA.

${ }^{7}$ Max-Planck-Institut für Physik, Munich, Germany.

${ }^{8}$ Department of Biological Sciences, University of Alberta, Edmonton, Alberta, Canada.

${ }^{9}$ Institut für Kernphysik, Goethe Universität, Frankfurt, Germany.

${ }^{10}$ Gesellschaft für Schwerionenforschung, Darmstadt, Germany. 